\title{
Der Kaffee in Landschaft und Wirtschaft Aethiopiens
}

\author{
Günther Schumacher
}

Noch vor kurzer Zeit war Aethiopien in Europa als kaffeeproduzierendes Land wenig bekannt. Infolge seiner Hochlandnatur nicht selten die «Schweiz Afrikas» genannt, besitzt es landwirtschaftlich jedoch überdurchschnittlich gute Möglichkeiten zur Gewinnung von Kaffee, dessen Ernte in den letzten Jahren zu einem besonderen Ereignis geworden ist, indem dieses Exportprodukt mit 50\% am Gesamtausfuhrwert des Landes beteiligt ist. Der Kaffee wurde somit zum wichtigsten devisenbringenden Ausfuhrerzeugnis. Die Jahresproduktion schwankt zwischen 40000 und 70000 Tonnen, was $2 \%$ der Welterzeugung und 20\% derjenigen Afrikas entspricht. Etwa $90 \%$ der Ernte entfallen auf wildwachsende Kaffebäume; dabei gilt es festzuhalten, daß in Aethiopien keineswegs unbeschränkt gute Voraussetzungen für den Anbau von Kaffee bestehen. Um dessen Bedeutung für das ganze Land beurteilen zu können, sei versucht, den Kaffee und seine Wirtschaft im Zusammenhang mit Natur und Kultur der ihn erzeugenden Landschaften zu sehen und deren Auswirkung auf die innere ebenso wie die äußere Entwicklung des Landes darzustellen.

\section{Die natürlichen Gegebenheiten}

Das äthiopische Hochland umfaßt rund $38 \%$ der Landesfläche, die Kerngebiete des Lebens- und Wirtschaftsraums des Staates (Fig. 1) und zerfällt in das zentrale und das südöstliche Hochplateau. Beide werden durch den nördlichen afrikanischen Grabenbruch getrennt, zu dem sie mit Steilwänden von bis zu $1000 \mathrm{~m}$ Tiefe abfallen. Tiefeingeschnittene, oft canyonartige Täler wie jene des Blauen Nils (Abbai), des Atbara, Baro, Omo, Webi Schebeli unterteilen das Plateau in weitere, fast unbezwingbare Hochländer, die von den Eingeborenen Amba genannt werden. Sie waren bekanntlich eine der Hauptvoraussetzungen für die lange Bewahrung der Unabhängigkeit Aethiopiens und seiner koptischen Kultur.

Auf kurze, vor allem vertikale Distanz bestehen große Unterschiede der Lebensformen. Grundsätzlich werden drei Höhenzonen unterschieden: die Kolla $(0-1600 / 1700 \mathrm{~m})$, ein sehr heißes, niederschlagsarmes Gebiet; die Woina Dega (1600/1700 $-2400 / 2500 \mathrm{~m}$ ) oder gemäßigte Zone sowie die
Dega (über $2400 / 2500 \mathrm{~m}$ ), die als kühle Zone betrachtet wird.

\section{Die Woina Dega}

ist das Hauptanbaugebiet für Kaffee (Fig. 1). Hier herrschte in früheren Jahrhunderten die Rebkultur vor (Woina Dega $=$ Weinland), die jedoch durch Kriege und Krankheiten vernichtet wurde. Der Kaffeebaum dagegen behielt infolge seiner größeren Widerstandsfähigkeit seine Wachstumsgebiete bei und vermochte sie sogar zu vergrößern. Er ist indessen nicht in der ganzen Woina Dega anzutreffen, sondern hauptsächlich in den unteren Regionen dieser Höhenzone, in der sogenannten Niederen Woina Dega, der Höhenstufe von 1600/1700 bis $2000 / 2100 \mathrm{~m}$.

Auf die Bedeutung der Woina Dega für Aethiopien weist der Vergleich der Flächenanteile mit den Exportwerten hin, wobei sich die Angaben auf das Jahr 1960 beziehen:

$\begin{array}{lcc} & \text { Fläche in \% } & \text { Bedeutung in \% } \\ \text { Kolla } & 62 & 10 \\ \text { Woina Dega } & 22 & 80 \\ \text { Dega } & 16 & 10\end{array}$

In der Woina Dega verhalten sich die Temperaturen in bezug auf die Monats- und Jahresmittel ziemlich konstant (Fig. 2); die Schwankungen zwischen Tag und Nacht dagegen sind bedeutend und können 5 bis $26^{\circ} \mathrm{C}$ betragen. Auch im Vergleich der beiden Hochplateaus bleiben die Temperaturen der gleichen Höhenlage in der Woina Dega praktisch unverändert, während bei den Niederschlägen größere Unterschiede festzustellen sind. Das zentrale Hochplateau verzeichnet durchwegs über 1000 mm Jahresniederschlag; das südöstliche Hochplateau erreicht diesen Jahreswert nur in wenigen günstig gelegenen Gebirgsregionen.

Die Niederschläge sind in zwei Regenperioden vorherrschend: in einer sogenannten kleinen (März und April) und in einer großen (Juli bis Oktober, [Fig. 3]). Im Südosten und Südwesten sind sie bei nur wenigen Trockenmonaten auf das ganze Jahr verteilt, während sie in Nordäthiopien, wie Station Gondar (Fig. 3) deutlich zeigt, auf eine kurze, starke Niederschlagsperiode in den Sommermonaten 


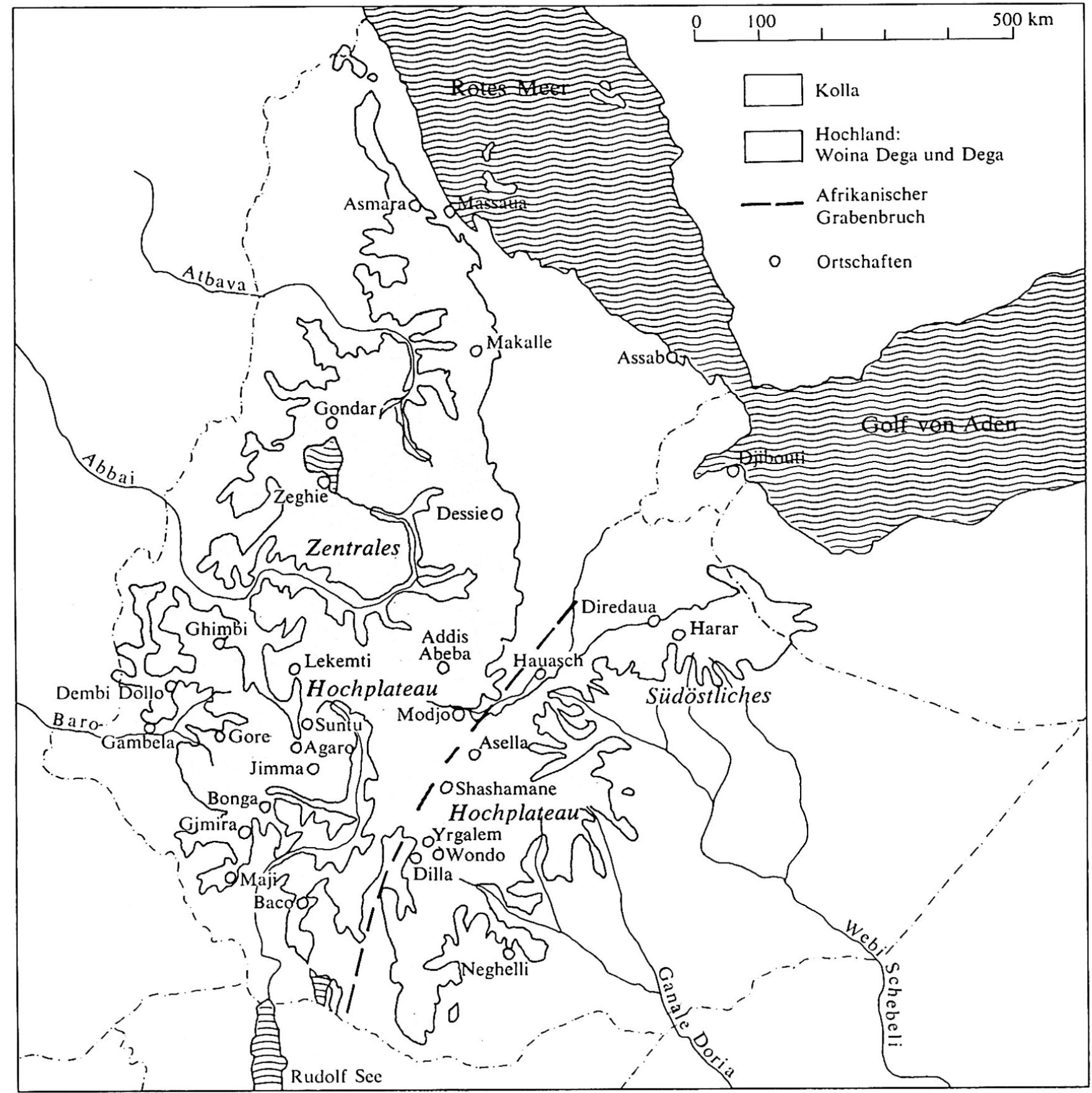

Fig. 1 Das äthiopische Hochland

beschränkt bleiben. Für die Kaffeepflanze sind ausgeglichene jahreszeitliche Niederschläge ein wichtiger Standortvorteil. Diese günstigen Voraussetzungen sind in den Provinzen Wollega, Ilu Babor, Kaffa-Jimma, Gamu-Gofa, Sidamo, Arussi und Harar (Fig. 4) gegeben, die 7 bis 10 humide beziehungsweise 2 bis 5 trockenere Monate aufweisen. Anderseits erfüllen die nördlichen Provinzen Beghemeder und Godjam diese Anforderungen mit 2 bis $4 \frac{1}{2}$ humiden beziehungsweise 10 bis $71 / 2$ trockeneren Monaten kaum. Für diese Niederschlagsschwankungen sind die Winde verantwortlich. Im Winter herrschen im gesamten Hochplateau die Nord- und Nordostmonsune vor; im Sommer teilt sich das Land in zwei Windbereiche auf. Das südöstliche
Hochplateau wird von den Süd- und Südostmonsunen überquert; das zentrale Hochland wird von Winden aus dem Westen beeinflußt, die vom Golf von Guinea her Aethiopien durchziehen.

Außer den klimatischen Faktoren ist der vulkanische und mineralreiche Boden für die Kaffeepflanze ein nicht zu übertreffender Standortvorteil; so wird dieser Boden oft auch «äquatorialer Löß» genannt und ist in mancher Beziehung mit den Bodengüten in den bekannten lateinamerikanischen Kaffeeanbaugebieten vergleichbar.

Bei den großen Höhenunterschieden im äthiopischen Hochland spielt die Erosion eine wichtige Rolle. Der oft ungeschützt an der Oberfläche liegende qualitativ gute Boden wird aus der nieder- 
schlagsreichen Woina Dega in die regenarme Kolla getragen. Besonders der Blaue Nil nimmt das für Aethiopien "wertvolle Exportgut» auf und befördert es in die Länder an seinem Unterlaufe.

Zusammenfassend können die natürlichen Gegebenheiten in der Niederen Woina Dega für den Kaffeeanbau in vielen Gebieten als geradezu ideal bezeichnet werden. Hier treffen die verschiedensten Vorzüge zusammen, wie es nur in wenigen Teilen der Erde der Fall ist: ein warmes, nicht zu heißes Klima, reiche jahreszeitliche Niederschläge und günstige Bodenverhältnisse.

\section{Der äthiopische Kaffee und sein Weg durch die Geschichte}

Eine erste Verwendung des Kaffees als Getränk scheint in engem Zusammenhang mit der Verbreitung des Islam zu stehen. Im Mittelalter und vermutlich auch schon früher wurde der Kaffee aus dem Innern des äthiopischen Hochlandes auf Karawanenwegen mühsam an die Küsten des Roten Meeres gebracht. Auf diese beschwerliche Wanderschaft weisen der heute in Aethiopien allgemein gebräuchliche Name für Kaffee "Buna» sowie die mohammedanischen Bezeichnungen "Kawah» oder "Kaweh» hin, die "aus Kaffa kommend», der heutigen Provinz Kaffa-Jimma, bedeuten. Später folgten Pflanzungen in besser zugänglichen Gebieten

\section{Temperaturdiagramme}

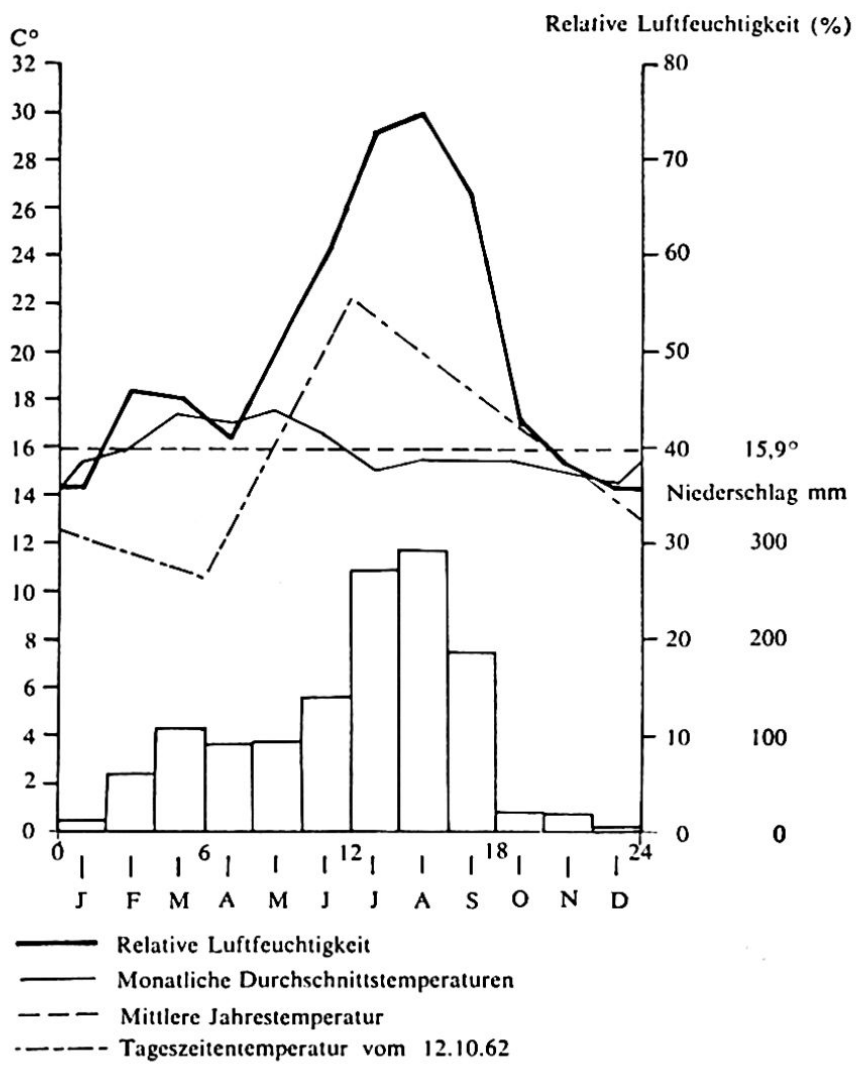

Fig. 2 Addis Abeba $2450 \mathrm{~m}$ ü. M. wie zum Beispiel Mocca, dem heutigen Yemen. So fiel das Ursprungsland des Kaffees allmählich der Vergessenheit anheim. Aus diesem Grunde wohl gab Carl Linné dieser Kaffeesorte die Bezeichnung "Coffea arabica» anstelle des ihr zukommenden Namens "Coffea abessinica».

Die erste neue, wichtige Verbindung Aethiopiens mit der Außenwelt erfolgte im Jahre 1917 durch die Eisenbahn von Djibouti über Diredaua nach Addis Abeba. Sie stellte zugleich den Anbruch der Moderne in dem bis dahin völlig in sich geschlossenen, konservativen Agrar- und Selbstversorgerstaate dar. Diese Selbstversorgung blieb bis $1935 \mathrm{mehr}$ oder weniger gewährleistet. Auch wurde der Handel eher gedrosselt, da mit Ausnahme des Kaffees den verlockenden Einfuhrprodukten äquivalente Ausfuhrgüter nicht gegenüberstanden. Doch besaß auch der Kaffee nur geringen Marktwert, und die äthiopische Sorte vermochte zu dieser Zeit mit dem

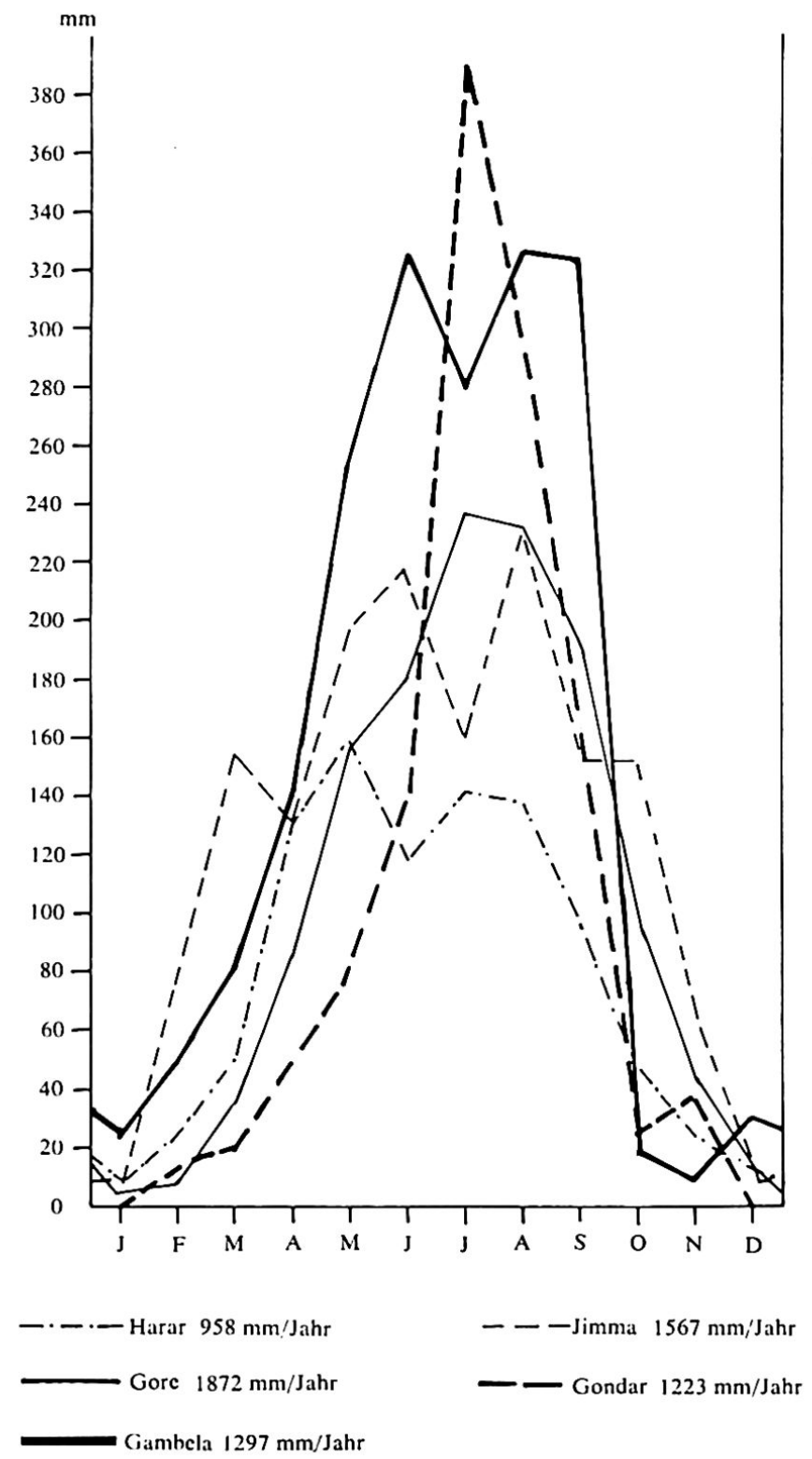

Fig. 3 Niederschlagsdiagramme einiger Kaffeehauptorte 


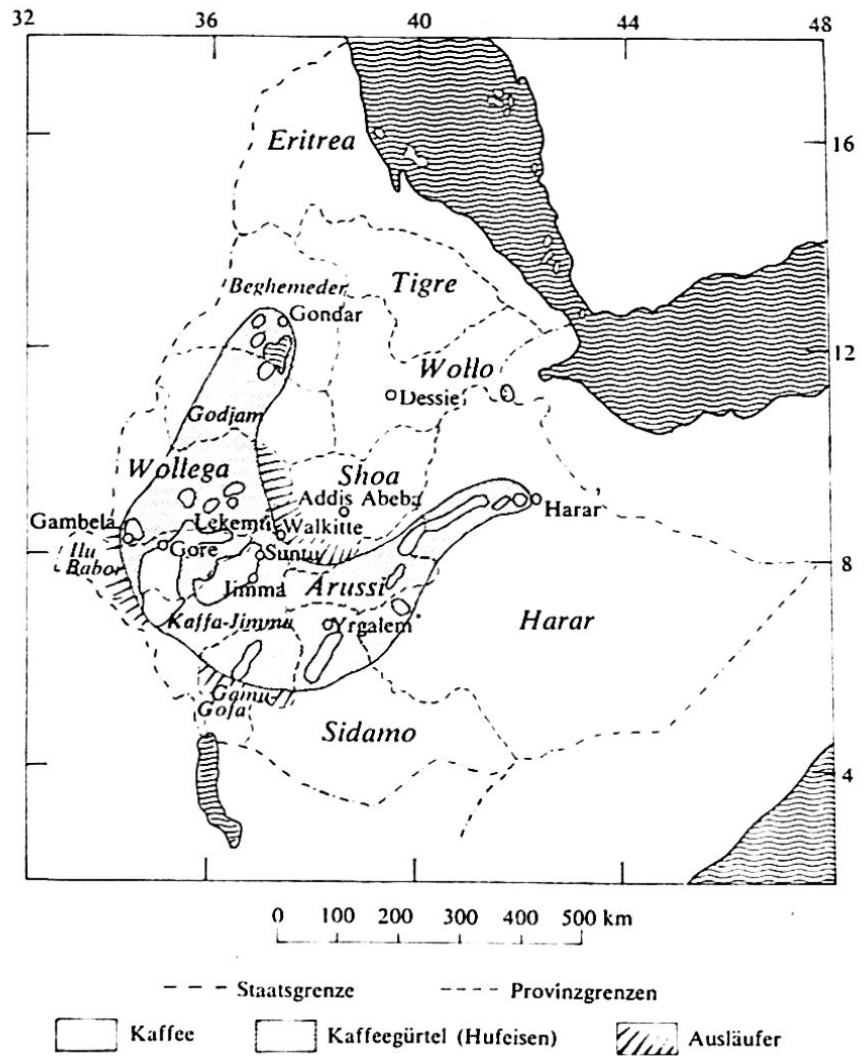

Fig. 4 Kaffeeanbaugebiete und Provinzen

«Tyrannen» Brasilien noch nicht zu konkurrieren. Erste Ansätze zur gelenkten Kaffeeproduktion und dessen Verarbeitung zeigten sich in den Jahren der Besetzung durch Italien, das mit der Schaffung vermehrter Verkehrswege den Zugang zu den Anbaugebieten erleichterte, Kraftwerke baute, den planmäßigen Anbau einführte, die erste Kaffeeverarbeitungsanlage und eine Sackfabrik errichtete. Nach dem Zweiten Weltkrieg nahmen sich die Vereinigten Staaten von Amerika als weltgrößter Kaffeeimporteur der Anbaugebiete Aethiopiens in zunehmendem $\mathrm{Maße}$ an, was einen merklichen Anstieg der Kaffeeproduktion und seines Exportes seit 1947 zur Folge hatte. Von besonderer Bedeutung für Aethiopien war das Jahr 1949, in welchem eine außerordentliche Mißernte in Brasilien vielen afrikanischen Anbaugebieten Impulse verlieh, diesem Weltmarktprodukt vermehrte Aufmerksamkeit zu schenken. Auf dieses "Sprungbett» legten die Aethiopier ihren Kaffee, der damit zum Hauptausfuhrerzeugnis wurde und in der Folge eine gewisse Monopolstellung erlangte. Von da an wurden jene Gebiete des wildwachsenden Kaffees intensiver genutzt, die bis anhin wegen zu großer Entfernung und $\mathrm{zu}$ hohen Transportkosten nur der Deckung des örtlichen Eigenbedarfs gedient hatten. Dadurch gelang es Aethiopien, bis 1952 mit rund 33500 Tonnen den vierten Rang der Kaffee-Exporteure Afrikas einzunehmen. In den letzten Jahren betrug die Exportquote bis zu 58500 Tonnen jährlich.

Eine exakte Übersicht über den von wildwachsenden Kaffebäumen einerseits und den auf Plantagen anderseits geernteten Kaffee ist kaum möglich, da die Anbaugebiete zu weit auseinanderliegen. Jedenfalls aber können die noch lange nicht in ihrer Gesamtheit erfaßten und erschöpften Wildkaffeebestände vorerst zu einem weiteren Produktions- und Exportanstieg beitragen.

Interessant für den Laien und für den Fachmann sind die drei Arten der natürlichen "Fortpflanzung» des Kaffees. Eine solche erfolgt zunächst durch das Austrocknen der Kaffekirsche am Baume. Wind oder Regen läßt diese zu Boden fallen, worauf die beiden im Fruchtfleisch befindlichen Bohnen nach ihrem Aufbrechen zu keimen beginnen. Eine andere Art ist das Fortpicken des Fruchtfleisches durch Vögel, wobei die Bohnen ebenfalls zu Boden fallen. Die dritte Fortpflanzungsart ist sowohl die sicherste als auch die merkwürdigste: Im Oberholz der Kaffeewälder halten sich mit Vorliebe ganze Horden von Goresa-Affen auf, die das süße, gutschmeckende Fruchtfleisch der Kaffeekirsche als Nahrung schätzen. Da aber die Kaffeebohnen bitter sind, werden sie von den Affen nicht zerbissen, sondern mitgeschluckt. Nach Austritt der Exkremente finden die Bohnen infolge der natürlichen Düngung beste Keimungsverhältnisse, und es kommt oft vor, daß auf ganz kleinem Raum mehrere Kaffeepflänzchen gleichzeitig um ihr Dasein kämpfen. Aufgrund dieser Fortpflanzungsweise ist es nicht erstaunlich, wenn in den dichten Wäldern auf einem Quadratmeter bis zu zwanzig Kaffeebäume wachsen, deren Ertrag jedoch gering ist. Durch Ausmerzung einiger dieser Bäume lassen sich aber vorzügliche Ernten erzielen, vor allem wenn die stehengebliebenen entsprechende Pflege erhalten.

Dem Plantagenkaffee wird ein Zehntel an der Gesamternte zugeschrieben. In den meisten Provinzen ist man jedoch dazu übergegangen, die wildwachsenden Bestände zu säubern und ertragarme Bäume durch eigens herangezogene und speziell ausgewählte Setzlinge zu ersetzen. Besonders in SüdwestAethiopien (Jimma, Sidamo, Arussi) wie auch in Harar ist diese Art des Kaffeeanbaus verbreitet, während in andern Gegenden kleinbäuerliche wildwachsende Bestände oder ungeordnetes, nicht zusammenhängendes Pflücken und Einbringen des Kaffees aus wildwachsenden Beständen anzutreffen sind. So ist es schwierig, einen durchschnittlichen Hektarertrag zu berechnen, der von wenigen bis zu hundert Kilogramm schwanken kann. Im Nachbarlande Kenya wurden Hektarerträge von bis zu $1000 \mathrm{~kg}$ erzielt, was auch für die zukünftigen Möglichkeiten in Aethiopien wegweisend sein kann. Zwischen wildem und Plantagenkaffee besteht 
grundsätzlich kein Qualitätsunterschied. Dagegen ergibt der Plantagenbetrieb größere Bohnen.

\section{Lage und Charakter der Anbaugebiete}

Die mit den Halbwild- und den Wildkaffeebeständen identischen Kaffeeanbaugebiete verlaufen in einem hufeisenförmigen Gürtel von einem nordwestlich gelegenen Zipfel bei Gondar (Tana-See) aus, nehmen gegen Südwesten an Breite bedeutend zu (Gambela-Sunto-Walkitte, zirka $300-400 \mathrm{~km}$ ) und verengen sich in nordöstlicher Richtung von Yrgalem an (Endpunkt Harar; Fig. 4). Entsprechend diesem Gürtel lassen sich Kern- und Nebenregionen unterscheiden.

Die Kern- und Idealgebiete setzen sich hauptsächlich aus Teilen der Provinzen Wollega, Ilu Babor, Kaffa-Jimma und Sidamo zusammen. Diese Kerngebiete können als die zentrale Achse des äthiopischen Kaffeeanbaues bezeichnet werden, da die Niederschläge ausreichend und auf das ganze Jahr verteilt sind (Fig. 3, Jimma). Als wichtigste Anbauorte sind zu nennen: Wollega: Lekemti, Ghimbi, Dembi-Dollo, Anfillo, Shebla, Nejeo, Asha. Ilu Babor: Gore, Mattu, Yagou, Doreni, Hurrumou. Kaffa-Jimma: Jimma, Agaro, Bonga, Wush-Wush, Gimira, Ghebi, Suntu, Manna, Mizan Tafari. Sidamo: Yrgalem, Dilla, Wondo-Kola, Yirga-Tscheffe, Tschelelektu, Agre-Selam, Lekku.

Die weniger begünstigten "Nebengebiete» unterscheiden sich zunächst durch die Lage, ferner durch die verschieden gelagerten Abweichungen von den Kerngebieten. Die nordwestlich des hufeisenförmigen Gürtels liegenden Teile der Provinzen Beghemeder und Godjam sind durch fast ausschließlich in den Sommermonaten auftretende Niederschläge benachteiligt, so daß die Kaffeepflanze während der anderen Monate an Wassermangel leidet (Fig. 3, Gondar). Die Gebiete der Provinzen Shoa und Gamu-Gofa, in denen ein guter Anbau noch möglich ist oder wäre, sind größtenteils als Ausläufer der Kerngebiete zu betrachten; die natürlichen Idealverhältnisse von Klima und Boden werden jedoch bereits stark vermindert. Die Provinzen Arussi und Harar bilden die Nebengebiete auf dem nordöstlichen Ausläufer des erwähnten Gürtels. Geringere Jahresniederschläge heben diese beiden Provinzteile von den andern genannten Gebieten ab. Die Verteilung der Niederschläge erfolgt prozentual fast wie in den Kerngebieten; doch reichen sie oft nicht mehr aus, um Wachstum und Gedeihen des Kaffeebaumes besonders in niederschlagsärmeren Jahren zu sichern. Aus diesem Grunde muß mit variierenden Ernteerträgen gerechnet werden, weshalb an verschiedenen Orten

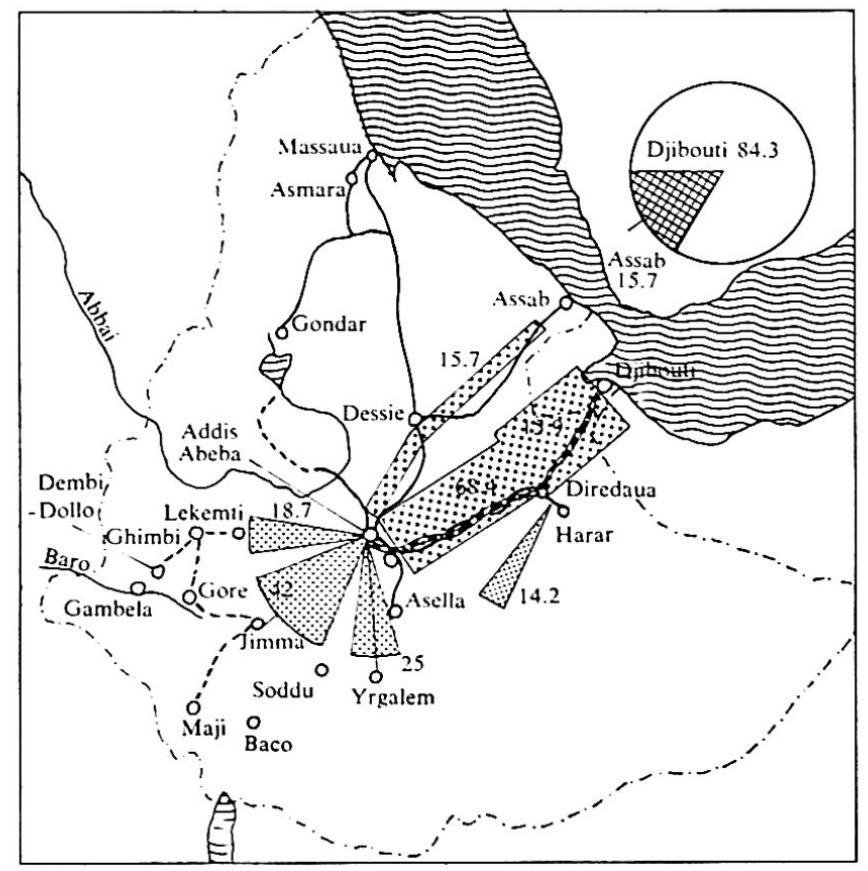

1 Mit Prozenten: von den Erzeugungsgürteln in die Zentren

$\because$ Mit Prozenten: von den Zentren in die Häfen (nach Zolldeklarationen)

Wichtige Handelsstraßen

-.- Pisten oder schlechte Verkehrsmöglichkeiten

$=-$ - Französisch-äthiopische Eisenbahn

\section{Fig. 5 Transportwege des Kaffees}

bereits mit Bewässerungsanlagen nachgeholfen wird.

In allen Kerngebieten ist der wildwachsende Kaffee mit dem weitaus größten Prozentsatz an der Ernte beteiligt. $\mathrm{Ab}$ und $\mathrm{zu}$ treten aber schon Plantagen auf, und große Teile gehören bereits den halbwilden Beständen an. Da in Zukunft nicht nur die Quantitäten, sondern vielmehr die Qualitäten entscheiden werden, dürften sich die Schwerpunkte immer mehr in die erwähnten Kerngebiete verlagern.

\section{Gründung und Aufgaben des National Coffee Board}

Im Jahre 1957 wurde der National Coffee Board ins Leben gerufen. Seither sind zahlreiche Koordinationsarbeiten durchgeführt worden. Sämtliche Behörden und Institute, die eine wirksame Tätigkeit in Zusammenhang mit dem Kaffee ausüben, wurden in dieser nationalen Kaffeebehörde vereinigt. Die Aufgabe des National Coffee Board läßt sich durch drei gesonderte Arbeitsbereiche umschreiben: den Field Service, der das Anlernen der einheimischen Bevölkerung im landwirtschaftlichen und vor allem im Kaffeesektor anstrebt; den technischen Service, dem der Unterhalt und die Ver- 
teilung des Maschinenparks obliegt; die administrativen Arbeiten. In enger Verbindung mit dem $\mathrm{Na}$ tional Coffee Board steht die Development Bank, deren Aufgabe es auch ist, den Bauern zinsgünstige Darlehen zu gewähren, welche für die Ausrüstung von Trockenplätzen und den Einkauf der wichtigsten Maschinen unentbehrlich sind.

\section{Kaffeepolitik und Kaffeehandel}

Neben den jeder Provinz eigenen Kaffeehaupt- und -sammelorten treten vor allem zwei Zentren in den Vordergrund: Addis Abeba (Makanissa) und Diredaua. Hier laufen die Fäden des ganzen Landes zusammen, wo der National Coffee Board auch seine Hauptkontrollorgane hat, da sich nicht jeder Kaffee für den Export eignet.

Der Kaffee ist eines der wenigen Produkte Aethiopiens, die mit einer Exportsteuer belegt werden. Eine Abgabe erfolgt unter anderem auch an den National Coffee Board. Die Inlandpreise für Kaffee wechseln je nach Erschließungsgrad und kaufmännischem Geschick der Bauern. In der Nähe der Allwetterstraßen wie in Dilla, Wondo, Jimma und Harar werden die höchsten Quoten erreicht. Die Bauern erhalten etwa $70-80 \%$ des Exportpreises, wenn sie nicht auf Unter- und Zwischenhändler angewiesen sind, die in der Regel wenig bezahlen. Diese meist arabischen Unterhändler bieten den Bauern 4 bis 5 äthiopische Dollar für eine Ferasula $(17 \mathrm{~kg})$, während sie in den Kaffeezentren 18 bis 21 Dollar dafür erzielen. Doch nicht nur diese Unterhändler ziehen Nutzen aus der Ignoranz und allfälligen Notlage der Bauern, sondern auch viele vorwiegend ausländische Exportfirmen, die den Kaffee aufgrund eines niedrigen Einheitspreises ankaufen. Doch gingen vor wenigen Jahren einige weitsichtige Firmen dazu über, den Bauern angemessene Preise zu offerieren. Jene Bauern, die keinen Anteil an einer ausgebauten Straße haben, sondern in weit abgelegenen Gebieten ihren Kaffee sammeln und verarbeiten, wie in Mizan Tafari, Tepi, Maji und Baco, müssen sich nach wie vor mit sehr tiefen Preisen abfinden.

\section{Transport des Kaffees vom Ursprungsort zu den Häfen}

Durch beschwerliche Überlandtransporte erleiden die landwirtschaftlichen Güter, darunter besonders der Kaffee, mehr oder weniger große Verteuerungen. Die Transportschwierigkeiten beginnen im Erzeugungsgebiet, da viele Pisten und Wege mit Lastwagen nicht mehr befahren werden können, sobald die Regenperiode anbricht. Dadurch ergibt sich eine sehr kurze Spitze für den Abtransport in jene lokalen Zentren, die über Lagerhallen verfügen und an ganzjährig befahrbare Straßen angeschlossen sind.

Die Straßenverbindung nach Assab, dem äthiopischen Hafen am Roten Meer, läßt viele Wünsche offen, da sie durch unwegsames Wüstengebiet führt. Die beste Verbindung nach außen stellt noch immer die Eisenbahnlinie nach Djibouti dar, die wieder mehr in den Interessenbereich des Landes gerückt ist, nachdem $50 \%$ der Aktien in äthiopischen Besitz übergegangen sind. In den Jahren 1960 und 1961 wurden über Assab jährlich durchschnittlich $15,7 \%$, über Djibouti $84,3 \%$ Kaffee ausgeführt (Fig. 5). Ein weiterer Ausfuhrhafen ist Gambela (Fig. 1) am Baro-Fluß. Dort aber müssen die Waren bei Beginn der Regenzeit eingetroffen sein, da der Fluß nur von Juli bis Oktober schiffbar ist. Stromabwärts führt der Weg zum Weißen Nil in den Sudan und nach Aegypten, wohin jährlich rund 200-500 Tonnen Kaffee exportiert werden.

\section{Die Exportländer}

Von den zirka 54000 Tonnen ausgeführten Kaffees des Jahres 1960 entfielen etwa $60 \%$ auf die USA, wo der verhältnismäßig billige und qualitativ noch nicht konkurrenzfähige äthiopische Kaffee meist zu Pulverkaffee verarbeitet wird. Hieraus erklärt sich auch die relativ kleine Kaffee-Exportquote nach Europa, wo die EWG-Staaten 1960 nur rund $11 \%$, die EFTA-Länder 7,6\% aufnehmen. Die äthiopischen Kaffee-Exporte konnten von 11883 Tonnen im Jahre 1943/44 auf 27503 Tonnen im Jahre 1950/51, auf 32953 Tonnen im Jahre 1955/56 und auf 58500 Tonnen im Jahre 1960/ 1961 gesteigert werden.

Im Verlaufe der letzten Jahre ist es Aethiopien gelungen, in mehreren europäischen Kaffeeverbraucherstaaten Absatzgebiete zu erschließen, wodurch das Handelsbilanzdefizit mit Europa einigermaßen tragbar wird. Die rund $60 \%$ des äthiopischen Kaffees aufkaufenden USA sind mit nur $15 \%$ am gesamten Importwert Aethiopiens beteiligt, während auf die europäischen Staaten etwa $45 \%$ entfallen.

\section{Die Zukunft des äthiopischen Kaffeeanbaues}

Auf dem heute bebauten Boden ließe sich mit Hilfe moderner Arbeitsmethoden eine drei- bis fünffache Steigerung der Kaffee-Erträge erzielen. Die Qualität könnte sogar zu einer der besten der Welt werden, was sich durch Erfolge auf kleineren Planta- 
gen, durch den Kaffeeanbau in Kenya und durch natürliche Gesundheit und Widerstandskraft in den Höhenlagen erweist. Von verschiedenen Institutionen des In- und Auslandes gingen Anregungen aus zur besseren Gestaltung der Betriebsgrößen für Kaffee und andere Kulturen sowie zur Regelung des Landbesitzes (Feudalsystem!).

Nach außen ist Aethiopien heute ein Kaffeemonopolland. Im Landesinnern ist es aber fehl am Platze, von einem monokulturellen Anbau zu sprechen, da Aethiopien über viele andere landwirtschaftliche Kulturen verfügt, die allerdings keine oder nur geringe Exportquoten aufweisen. Die «Schweiz Afrikas» ist ein Selbstversorgerstaat, der nur $10 \%$ seiner übrigen agrarischen Güter exportiert. Dadurch ist Aethiopien den Schwankungen auf dem Weltkaffeemarkt weniger ausgesetzt als andere bekannte Kaffee anbauende Länder.

\section{Résumé}

\section{La culture du café et son rôle dans l'économie d'Ethiopie}

L'Ethiopie, nommé aussi «la Suisse de l'Afrique», était jusqu'à nos jours presque inconnu comme pays producteur et exportateur de café. La production annuelle a varié pendant les dernières années entre 40000 et 70000 t de café, ce qui représente à peu près $2 \%$ de la production mondiale ou $20 \%$ de la production africaine. Le $90 \%$ du café est produit par des caféiers non cultivés formant une partie du sous-bois des forêts. Les conditions physiques sont très favorables aux plantes caféières. La zone d'altitude entre $1600 / 1700-2400 / 2500 \mathrm{~m}$, qu'on nomme la Woina Dega, favorise idéalement dans sa partie inférieure (1600/1700-2000/2100 m) cette culture. Le caféier exige approximativement $1000-1500 \mathrm{~mm}$ de précipitation annuelle, répartie équitablement tout au long de l'année (7-10 mois humides, $2-5$ mois secs). Les régions favorisées se situent dans les provinces de Wollega, Ilu Babor, Kaffa-Jimma et Sidamo; les parties des régions des provinces de Harar, Arussi, Gamu-Gofa, Shoa, Beghemeder et Godjam ne profitent plus des conditions idéales. La crise de production du Brésil, en 1949, a provoqué une forte expansion de l'exportation. Depuis 1957 existe le National Coffee Board (Ministère de Café), qui surveille et organise l'information des paysans, la production et l'exportation. Le port de Djibouti (Somalie française) est le principal port d'exportation (84\%) et le port éthiopien de Assab expédie 16\%. Le principal acheteur de café éthiopien était, en 1960, les Etats-Unis $(60 \%)$, puis la CEE $(11 \%)$ et l'AELE (7,6\%).

Il sera possible d'augmenter 3 ou 5 fois la production par une meilleure utilisation du sol et par une amélioration des installations mécaniques. 